\title{
Optimal first trimester preeclampsia prediction: a comparison of multimarker algorithm, risk profiles and their sequential application
}

\author{
R. Gabbay-Benziv', N. Oliveira ${ }^{2}$ and A. A. Baschat ${ }^{3 *}$ \\ 'Helen Schneider Hospital for Women, Rabin Medical Center, PetachTikva; Sackler Faculty of Medicine, Tel Aviv University, Tel Aviv, Israel \\ 2Department of Obstetrics and Gynecology, Maternidade Dr. Alfredo da Costa, Lisbon, Portugal \\ ${ }^{3}$ Department of Gynecology and Obstetrics, Johns Hopkins School of Medicine, Baltimore, MD, United States \\ *Correspondence to: Ahmet A. Baschat. E-mail: abaschal@jhmi.edu
}

\begin{abstract}
Objective To compare performance of multimarker algorithm, risk profiles and their sequential application in prediction of preeclampsia and determining potential intervention targets.
\end{abstract}

Study Design Maternal characteristics, ultrasound variables and serum biomarkers were collected prospectively at first trimester. Univariate analysis identified preeclampsia associated variables followed by logistic regression analysis to determine the prediction rule. Combined characteristics of the cardiovascular, metabolic and the personal risk factors were compared to the multimarker algorithm and the sequential application of both methods.

Results Out of 2433 women, 108 developed preeclampsia (4.4\%). Probability scores considering nulliparity, prior preeclampsia, body mass index, diastolic blood pressure and placental growth factor had an area under the receiver operating characteristic curve $0.784(95 \% \mathrm{CI}=0.721-0.847)$. While the multimarker algorithm had the lowest false negative rate, sequential application of cardiovascular and metabolic risk profiles in screen positives reduced false positives by $26 \%$ and identified blood pressure and metabolic risk in 49/54 (91\%) women with subsequent preeclampsia as treatable risk factors.

Conclusion Sequential application of a multimarker algorithm followed by determination of treatable risk factors in screen positive women is the optimal approach for first trimester preeclampsia prediction and identification of women that may benefit from targeted metabolic or cardiovascular treatment. (C) 2015 John Wiley \& Sons, Ltd.

Funding sources: Diagnostic Technologies LTD and PerkinElmer

Conflicts of interest: None declared

\section{INTRODUCTION}

Pre-eclampsia (PE) is a multisystem disorder that complicates $2-7 \%$ of all pregnancies and is the most important contributor to both adverse maternal and fetal outcomes. ${ }^{1,2}$ While abnormal placentation is a major factor in the pathophysiology of PE it has been long recognized that there are multiple contributors that determine a woman's risk to develop this complication. ${ }^{3,4}$ Evidence has been increasingly accumulated that prevention is most effective if initiated by the second trimester thereby directing efforts to identify methods that can predict PE risk in the first trimester. ${ }^{4-7}$ This research has resulted in the development of several prediction rules that incorporate multiple risk factors to derive individualized probabilities for $\mathrm{PE}$. The potential disadvantages of first trimester prediction rules include low positive predictive values (PPVs), concerns about their external validity and the inability to determine potential treatment targets for prevention. ${ }^{4,8}$

Previous studies have linked the development of PE to distinct risk profiles in women. These include the cardiovascular profile defined by hypertension or latent hypertension ${ }^{9-11}$; the metabolic profile defined by WHO criteria $^{12,13}$ and the thrombotic risk profile. ${ }^{14,15}$ A recent study demonstrated that at least one of these risk profiles is present in almost $80 \%$ of women that developed PE. ${ }^{16}$ Moreover, almost all reported first trimester PE screening algorithms utilize variables that reflect one of these three risk profiles. ${ }^{4}$ Accordingly, first trimester prediction of $\mathrm{PE}$ by risk profiles alone may be as accurate as utilizing more complex multimarker prediction rules and may have the added advantage in identifying treatable risk factors. Thus, it was the aim of this study to compare the predictive performance of risk profiles alone, the multimarker prediction rule and their sequential application when applied to the same prospectively enrolled group of women.

\section{MATERIALS AND METHODS}

This is a secondary analysis of a prospective, observational study with the aim of developing a first trimester predictive model for PE. ${ }^{17}$ Women presenting with singleton gestation 
at 9-14 weeks were enrolled by informed written consent. A questionnaire was utilized to ascertain relevant medical history, and a standardized trans-abdominal ultrasound examination was performed to confirm gestational age, measure the fetal crown-rump length and perform uterine artery Dopplers to measure the Pulsatility Index. On maternal examination the weight (in $\mathrm{kg}$ ), height (in $\mathrm{cm}$ ) and body mass index (BMI, $\mathrm{kg} / \mathrm{m}^{2}$ ) were measured on regularly calibrated equipment. Blood pressure measurements ( $\mathrm{BP}, \mathrm{mmHg}$ ) were taken using the Dinamap Pro 1000 V3 (GE Medical Systems, Milwaukee, WI) automated sphygmomanometer, with a cuff size appropriate for maternal arm circumference. Sphygmomanometer calibration occurred every 6 months in accordance with the Association for the Advancement of Medical Instrumentation guidelines. Maternal blood samples obtained by occlusive venipuncture were analyzed for serum concentration of pregnancy-associated protein-A (PAPP-A), free beta human chorionic gonadotrophin (free $\beta$-HCG) and placental growth factor (PlGF). Pregnancy outcome was ascertained by study personnel and verified by source documentation.

PE was defined as new-onset or worsening proteinuria and systolic blood pressure $\geq 140 \mathrm{mmHg}$ and/or diastolic blood pressure $\geq 90 \mathrm{mmHg}$ on two separate occasions 6 or more hours apart after 20 weeks of gestation. PE superimposed on chronic hypertension was defined as worsening BP and increasing proteinuria after 20 weeks of gestation. Early PE was defined as PE requiring delivery $<34$ weeks and late PE defined as PE requiring delivery $\geq 34$ weeks of gestation. For this analysis we excluded patients that received aspirin prior to 16 weeks' gestation and patient with prothrombotic risk profiles receiving heparin for prevention of PE as they were already recognized at risk and treated accordingly.

Univariate analysis was performed to identify statistically significant individual factors that were associated with subsequent development of PE. Relevant key factors that were found statistically significant on the univariate analysis were subsequently stratified as cardiovascular or metabolic or personal risk modifiers. Continuous variables were transformed to categorical ones using receiver operator characteristics (ROC) statistics with Youden's Index as cut off values. Women were then assigned as positive (1) or negative (0) for every risk profile based on the relevant key factors. Next, we utilized all significant key variables together in logistic regression analysis to determine the best multimarker prediction algorithm for first trimester prediction of PE. The optimal probability score cut-off was determined using ROC curve with sensitivity set at $90 \%$. Women were assigned as screen positive or screen negative according to above prediction algorithm. Finally, we compared the predictive performance of each risk profile individually, the constructed multimarker algorithm and sequential application of the two strategies. True positive (TP), true negative (TN), false positive (FP) and false negative (FN) were calculated as well as risk profiles ability to identify treatable risk factors (cardiovascular or metabolic) among all screened positive women.

The statistical software package SPSS 14.0 (SPSS Inc. Chicago, IL, USA) was used for all data analyses, and a $P$ value of $<0.05$ was considered significant.

\section{RESULTS}

Of 2433 women meeting inclusion criteria, 108 (4.4\%) developed PE, 18 of these were early-onset PE. The mean maternal age was 29.5 \pm 6.5 years (range, 18-55) and 1063 $(43.7 \%)$ were nulliparous. The majority of women were of either Caucasian or African-Americans ethnicity $(43.3 \%$ and $49.8 \%$, respectively). Prior history was significant for chronic hypertension (156, 6.4\%), diabetes mellitus (86, 3.5\%), thrombophilia $(3,0.1 \%)$, prior $\mathrm{PE}(69,2.8 \%)$ and prior gestational diabetes $\left(44,1.8 \%\right.$ ). Mean BMI was $28.4 \mathrm{~kg} / \mathrm{m}^{2}$, and mean arterial BP was $83 \mathrm{mmHg}$ at enrollment. Delivery was at mean gestational age of $38.8 \pm 2.3$ weeks (range 20.6-42.6), and the mean birth weight was $3218 \pm 601 \mathrm{~g}$. One-hundred seventy three $(7.1 \%)$ women delivered infants with a birth weight above the $90^{\text {th }}$ percentile and $221(9.1 \%)$ with a weight below the $10^{\text {th }}$ percentile.

Table 1 presents first trimester maternal characteristics and clinical variables stratified by subsequent development of PE. Women with PE were more likely to be of Hispanic ethnicity, nulliparous, hypertensive and diabetic. They were more likely to have a prior history of PE, and at enrollment their BPs and BMIs were higher while PlGF (MoM) and PAPP-A values (MoM) were lower.

Statistically significant variables were grouped by their corresponding risk factors, and women were categorized as positive (1) or negative (0) accordingly. Chronic hypertension (listed as pre-pregnancy maternal diagnosis) and a BP over $120 / 71.5 \mathrm{mmHg}$ at first trimester (values calculated by ROC statistics) were identified as the significant factors defining the cardiovascular risk profile. Maternal diabetes, maternal BMI $>28.7 \mathrm{~kg} / \mathrm{m}^{2}$ or ovulation induction defined the metabolic risk profile. Finally, logistic regression analysis identified nulliparity and prior PE as the significant personal risk modifiers.

The first trimester multimarker algorithm for prediction of PE was derived from 1258 women with available placental biomarkers results. All variables identified as significant in the univariate analysis were entered into logistic regression analysis to construct a prediction rule for PE. The final model included nulliparity, prior PE, BMI, diastolic BP and PlGF, and the probability scores generated an area of 0.784 (95\% CI 0.721-0.847) under the curve on ROC curve statistics (Figure 1). A probability score of 0.021 cut-off value corresponded to $90 \%$ sensitivity, $40 \%$ specificity, $7 \%$ PPV and $99 \%$ negative predictive value (NPV) (Table 2).

Individually, all risk profiles as well as the multimarker algorithm significantly predicted $\mathrm{PE}(P=0.000$ for all). In the sequential approach, risk profiles were applied on the multimarker algorithm screened positive women, and only the cardiovascular and metabolic risk profiles remained as significant risk stratifiers $(P=0.019$ and 0.016 , respectively, Table 2). As cardiovascular and metabolic risk profiles have specific treatments they were included in the sequential approach to identify the number of women that may benefit from targeted treatment based on their risk profile. Among 771 screen positive women, the algorithm identified 54 TP women with subsequent development of PE. Applying risk profiles in the sequential approach further identified $49 / 54$ (90.1\%) patients as having risk profiles (cardiovascular or metabolic) that can be 
Table 1 First trimester maternal characteristics and clinical measurements stratified by subsequent development of pre-eclampsia

\begin{tabular}{|c|c|c|c|}
\hline Maternal characteristic and clinical variables & Developed PE N= 108 & Rest of cohort $N=2325$ & $P$ value \\
\hline Maternal age (years), mean \pm SD & $28.7 \pm 7.2$ & $29.6 \pm 6.5$ & 0.158 \\
\hline Nulliparity N (\%) & $66(61.1)$ & $997(42.9)$ & 0.000 \\
\hline Smoking N (\%) & $11(10.2)$ & $228(9.8)$ & 0.869 \\
\hline \multicolumn{4}{|l|}{ Ethnicity N (\%) } \\
\hline White & $38(35.2)$ & $1016(43.7)$ & 0.091 \\
\hline African-American & $62(57.4)$ & $1150(49.5)$ & 0.116 \\
\hline South Asian & $3(2.8)$ & $89(3.8)$ & 0.797 \\
\hline Hispanic & $4(3.7)$ & $27(1.2)$ & 0.046 \\
\hline Others & $1(0.9)$ & $43(1.8)$ & 0.721 \\
\hline History of hypertension N (\%) & $24(22.2)$ & $132(5.7)$ & 0.000 \\
\hline History of diabetes $N(\%)$ & $14(13)$ & $72(3.1)$ & 0.000 \\
\hline History of nephropathy N (\%) & $1(0.9)$ & $1(0.0)$ & 0.087 \\
\hline History of thrombophilia N (\%) & $0(0)$ & $3(0.1)$ & 0.873 \\
\hline History of PE N (\%) & $12(11.1)$ & $57(2.5)$ & 0.000 \\
\hline History of gestational diabetes $N(\%)$ & $2(1.9)$ & $42(1.8)$ & 0.590 \\
\hline Ovulation induction $N(\%)$ & $2(1.9)$ & $14(0.6)$ & 0.157 \\
\hline First trimester $B M I\left(\mathrm{~kg} / \mathrm{m}^{2}\right)$, mean $\pm S D$ & $31.0 \pm 9.2$ & $28.2 \pm 7.2$ & 0.002 \\
\hline \multicolumn{4}{|l|}{ Obesity level $N(\%)$} \\
\hline$<20 \mathrm{~kg} / \mathrm{m}^{2}$ & $6(5.6)$ & $138(5.9)$ & \\
\hline $20-25 \mathrm{~kg} / \mathrm{m}^{2}$ & $29(26.9)$ & $769(33.1)$ & \\
\hline $25-30 \mathrm{~kg} / \mathrm{m}^{2}$ & $23(21.3)$ & $354(15.2)$ & \\
\hline $30-35 \mathrm{~kg} / \mathrm{m}^{2}$ & $15(13.9)$ & $235(10.1)$ & \\
\hline $35-40 \mathrm{~kg} / \mathrm{m}^{2}$ & $15(13.9)$ & $162(7)$ & \\
\hline$\geq 40 \mathrm{~kg} / \mathrm{m}^{2}$ & $20(18.5)$ & $667(28.7)$ & 0.008 \\
\hline \multicolumn{4}{|l|}{ First trimester SBP N (\%) } \\
\hline$<120 \mathrm{mmHg}$ & $35(32.4)$ & $1605(69)$ & \\
\hline $120-140 \mathrm{mmHg}$ & $60(55.6)$ & $655(28.2)$ & \\
\hline$\geq 140 \mathrm{mmHg}$ & $13(12)$ & $65(2.8)$ & 0.000 \\
\hline \multicolumn{4}{|l|}{ First trimester DBP } \\
\hline$<80 \mathrm{mmHg}$ & $84(77.8)$ & $2166(93.2)$ & \\
\hline $80-90 \mathrm{mmHg}$ & $18(16.7)$ & $143(6.2)$ & \\
\hline$\geq 90 \mathrm{mmHg}$ & $6(5.6)$ & $16(0.7)$ & 0.000 \\
\hline MAP $(m m H g)$, mean \pm SD & $92 \pm 10$ & $83 \pm 8$ & 0.000 \\
\hline PAPP-A (MoM), mean \pm SD & $1.0 \pm 0.7$ & $1.2 \pm 0.7$ & 0.000 \\
\hline $\mathrm{PIGF}(\mathrm{M} \circ \mathrm{M})$, mean $\pm \mathrm{SD}$ & $0.9 \pm 0.4$ & $1.1 \pm 1.2$ & 0.004 \\
\hline Mean UtA PIs (MoM), mean \pm SD & $1.1 \pm 0.4$ & $1.1 \pm 0.4$ & 0.339 \\
\hline
\end{tabular}

BMI, body mass index; SBP, systolic blood pressure; DBP, diastolic blood pressure; PAPP-A, placental associated plasma protein A; HCG, human chorionic gonadotrophin; PIGF, placental growth factor; UtA PI, uterine artery pulsatility index; MoM, multiple of medians; MAP, mean arterial blood pressure.

treated in an individual manner. Moreover, the multimarker algorithm itself identified 717 women as FP. Applying risk profiles identified $188 / 717$ (26.2\%) of them as FP thereby and therefore not in need for further intervention.

\section{DISCUSSION}

First trimester screening algorithms contain a combination of maternal, placental and biophysical variables to derive individualized risk for PE with little guidance to the treatment that is most appropriate to prevent disease. ${ }^{4,18}$ As PE is more frequent in women with a defined set of risk profiles with potential treatments, we evaluated if these are of added utility in risk stratification. Our study demonstrates that sequential application of a multimarker algorithm followed by risk profile categorization in screen positive women numerically provides the best prediction of PE. This sequential approach lowered false positive rate by over $25 \%$ and halved the false negative rate. Moreover, risk profiles identified management of cardiovascular or metabolic risk factors as potential treatment goals in over $90 \%$ of women that subsequently developed PE. 


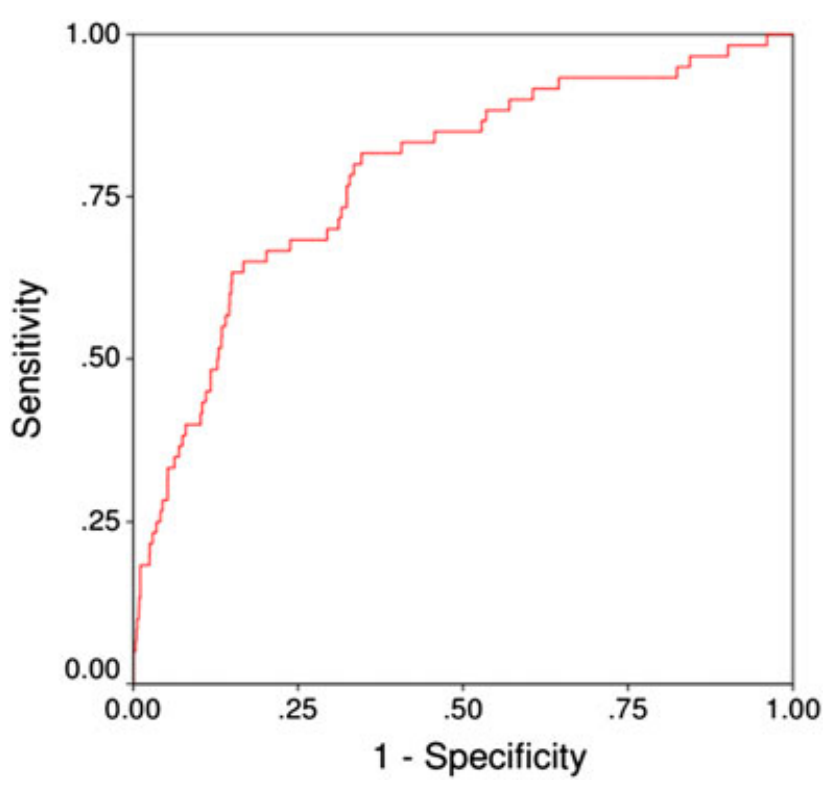

Figure 1 Prediction performance of multimarker algorithm for first trimester prediction of pre-eclampsia. Area under the curve is 0.784 (95\% Cl 0.721-0.847)

Previous studies have shown that multimarker algorithms as well as individual risk profiles are effective in identifying women at risk for PE. ${ }^{17,19-25}$ The variable prevalence of cardiovascular, metabolic and prothrombotic risk profiles across populations appears to be a limiter to their generalized screening application. ${ }^{8,16,26,27}$ Multimarker algorithms on the other hand also incorporate placental factors and through mathematical consideration of a wider range of risk modifiers are able to provide better risk stratification in their populations of origin. ${ }^{4,5,8,22,26}$ Presently, women identified at risk for PE are offered first trimester low-dose aspirin based on the recommendations of the US Preventive services task force. ${ }^{7}$ However, the benefits of low dose aspirin may be limited to women with a high prevalence of cardiovascular and metabolic risk profiles and a low prevalence of prothrombotic risks. ${ }^{18,28}$ Accordingly, aspirin alone appears to address a specific risk profile if initiated by 16 weeks but has limited utility as a universal prevention for all women defined at risk by different prediction strategies. ${ }^{6,7}$ This raises the question if additional therapies could be offered to women where a sequential screening approach identifies additional risk profiles.
Observations in women with polycystic ovarian syndrome that become pregnant suggests that continuation of Metformin is associated with reduced rates of PE which appear to be partly mediated by accelerated normalization of first trimester uterine artery blood flow resistance. ${ }^{29-32}$ Pravastatin inhibits sFLT- 1 release in mouse models and is currently being evaluated in two human randomized controlled clinical trials for their safety in pregnancy and their efficacy in the prevention of PE (www.controlled-trials.com: ISRCTN23410175 and ClinicalTrials.gov: NCT01717586). ${ }^{33-36}$ It is therefore likely that evidenced based recommendations will become available in the near future to offer women with a metabolic risk profile identified at first trimester sequential PE screening risk specific preventive therapy in addition to low dose aspirin. ${ }^{4}$ Hypertension is a key component of the cardiovascular and metabolic risk profile and accordingly numerically the most important first trimester contributor to PE risk. Unfortunately, opinions regarding the optimal treatment threshold in the first trimester diverge strongly. The risk for hypertensive complications increases at blood pressures that are below the treatment threshold that is currently recommended by most professional societies. ${ }^{4,37-}$ ${ }^{39}$ Accordingly, research to define the appropriate approach to blood pressure in first trimester screen positive women for prevention of PE is urgently needed. Therefore application of a sequential first trimester screening approach for $\mathrm{PE}$ would enable administration of risk specific therapy to women with metabolic risks and provide a tool to define women that are appropriate candidates for research studies on the appropriate first trimester initiation of blood pressure management. We suggest a two-step screening protocol in which a multimarker algorithm will be applied first, in order to detect women at risk for future PE with the highest sensitivity, followed by individualized treatment based on stratification by risk profiles (Figure 2).

The strengths of our study lie in the large number of prospectively enrolled patients with outcomes that were verified with precise source documentation. The prevalence of the individual risk profiles and PE allowed us to test our hypothesis. Our data suggests that the optimal screening approach lies in the development of sequential screening algorithm where the first step should be optimized to offer the highest sensitivity (for this purpose incorporation of additional prothrombotic markers such as serum homocysteine as well as modification of statistical

Table 2 Prediction performance of multimarker algorithm and risk profiles for subsequent development of pre-eclampsia-individual versus sequential approach

\begin{tabular}{|c|c|c|c|c|c|c|c|c|}
\hline & Sensitivity & Specificity & PPV & NPV & & & & \\
\hline & $\%(95 \% \mathrm{Cl})$ & $\%(95 \% \mathrm{Cl})$ & $\%(95 \% \mathrm{Cl})$ & $\%(95 \% \mathrm{Cl})$ & $\mathrm{TP}$ & $\mathrm{TN}$ & FP & FN \\
\hline Cardiovascular risk profile ( $N=2433$ ) & $80.6(71.8-87.5)$ & $59.2(57.2-61.2)$ & $8.4(6.8-10.3)$ & $99(97.7-99.1)$ & 87 & 1376 & 949 & 21 \\
\hline Metabolic risk profile $(N=2433)$ & $60.6(50.7-69.8)$ & $61.9(59.9-63.9)$ & $6.9(5.4-8.7)$ & $97.1(96.1-97.9)$ & 66 & 1439 & 886 & 42 \\
\hline Personal risk profile ( $N=2433$ ) & $71.3(61.8-79.6)$ & $54.7(52.7-56.8)$ & $6.8(5.4-8.4)$ & $97.6(96.6-98.4)$ & 77 & 1272 & 1053 & 31 \\
\hline Multimarker algorithm ( $N=2433$ ) & $90(79.5-96.2)$ & $40.2(37.4-43.0)$ & $7(5.3-9)$ & $98.8(97.3-99.5)$ & 54 & 481 & 717 & 6 \\
\hline Second stage sequential analysis ${ }^{a}(N=771)$ & $90.7(79.7-96.9)$ & $26.2(23-29.6)$ & $8.5(6.3-11.1)$ & $97.4(94.1-99.1)$ & 49 & 188 & 529 & 5 \\
\hline
\end{tabular}

PPV, positive predictive value; NPV, negative predictive value; TP, true positive; TN, true negative; FP, false positive; FN, false negative.

${ }^{a}$ Sequential approach - this line refers to applying cardiovascular or metabolic risk profiles (treatable) only on women screened positive by the multimarker algorithm. 


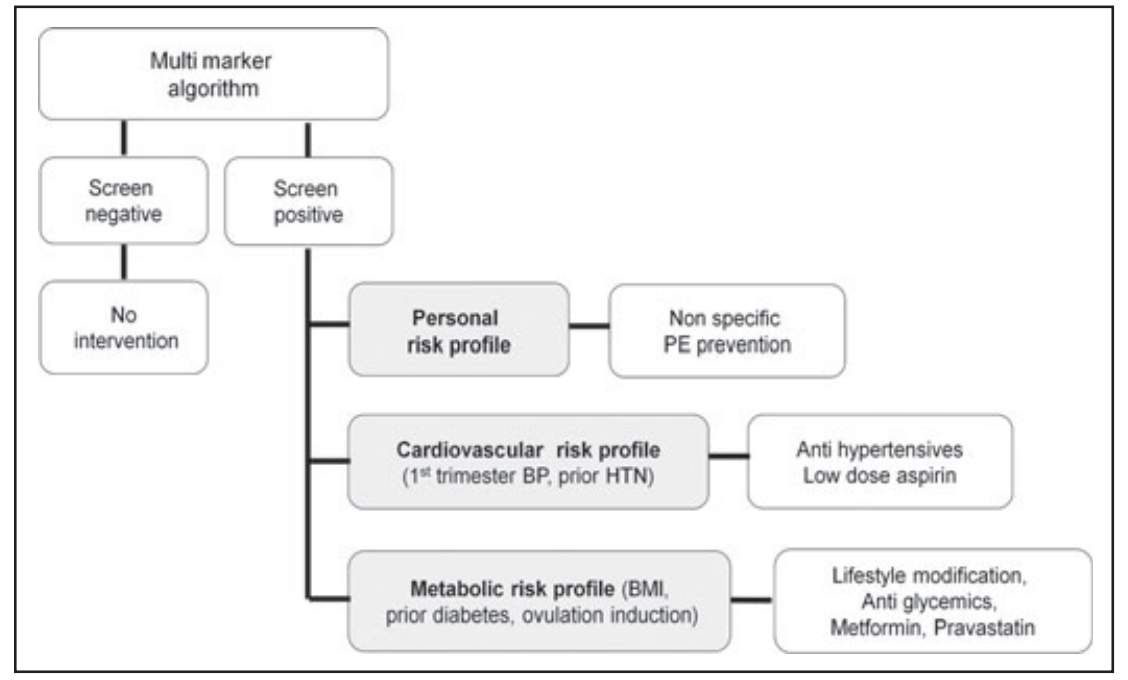

Figure 2 Suggested optimal approach for first trimester prediction of pre-eclampsia

approaches may prove useful ${ }^{4,5,16,19,21,40}$ ) and the second step will identify the appropriate threshold for cardiovascular, metabolic and prothrombotic risk profiles to trigger intervention. This is most tangible for metabolic and prothrombotic risks and controversial for defining blood pressure treatment targets for patients identified with cardiovascular risk in the first trimester. Given the prevalence and relevance of the cardiovascular risk profile in pregnancy outcome and long-term health of women, sequential first trimester PE screening algorithms may prove to be critical to advance research in this area of women's healthcare. Nevertheless, our study has some limitations to consider: because of their documented importance in determining the accuracy of multimarker algorithms, we chose to include placental biomarkers results in our study. As these were only available for a subset of study participants this limited our sample size for the sequential prediction approach. However, we chose to use the entire cohort in order to obtain better accuracy for the individual risk factors in the univariate analysis. Also, this limited sample size prevented us from splitting it to a training group and a validation group. This will need to be done in future greater samples. Moreover, the exclusion of women receiving aspirin and anticoagulation further skewed our population towards women with cardiovascular and metabolic risk profiles, which are prevalent in the environment where this study was conducted. Finally, we pre-determined that for the sequential approach the multimarker algorithm was applied before the risk profiles. This approach was chosen based on a clinical model where women that are screened positive would be further evaluated for specific treatment.
In summary, we present a new sequential approach for first trimester prediction of PE using a multimarker algorithm followed by application of risk profiles. This approach correctly predicts the highest proportion of women that develop PE and has the advantage of identifying potential treatment targets to prevent PE. This sequential screening approach may prove beneficial to determine women who should receive management for metabolic risks and to clarify appropriate management of cardiovascular risks.

\section{WHAT'S ALREADY KNOWN ABOUT THIS TOPIC?}

- First trimester prediction of preeclampsia is feasible by multimarker algorithms incorporating maternal characteristics, ultrasound variables and serum biomarkers or by stratifying women based on their risk profile: mainly thrombotic, cardiovascular or metabolic.

- The optimal method of prediction is still unknown.

- All women at high risk for developing preeclampsia are treated the same by low dose aspirin.

\section{WHAT DOES THIS STUDY ADD?}

- Combination of multimarker algorithm followed by further stratification of the screen positive women by risk profiles has two advantages: (a) lowering false positive rate, therefore preventing unnecessary treatment and (b) enabling the potential for targeted treatment based on the individual risk profile (i.e. metabolic, cardiovascular or thromboticl.

\section{REFERENCES}

1. Sibai B, Dekker G, Kupferminc M. Pre-eclampsia. Lancet 2005;365: 785-99.

2. American College of Obstetricians and Gynecologists. Diagnosis of Management of Pre-Eclampsia and Eclampsia, ACOG Practice Bulletin \#33. American College of Obstetricians and Gynecologists: Washington, DC; 2002.

3. Chesley LC. History and epidemiology of preeclampsia-eclampsia. Clin Obstet Gynecol 1984;27:801-20.
4. Baschat AA. First trimester screening for pre-eclampsia: moving from personalized risk prediction to prevention. Ultrasound Obstet Gynecol 2015;45:119-29.

5. Nicolaides KH. Turning the pyramid of prenatal care. Fetal Diagn Ther 2011;29:183-96.

6. Bujold E, Roberge S, Lacasse Y, et al. Prevention of preeclampsia and intrauterine growth restriction with aspirin started in early pregnancy: a meta-analysis. Obstet Gynecol 2010;116:402-14. 
7. Levefre ML. Low-dose aspirin use for the prevention of morbidity and mortality from pre-eclampsia: U.S. Preventive Services Task Force Recommendation Statement. Ann Intern Med 2014;161:819-26.

8. Oliveira N, Magder LS, Blitzer MG, Baschat AA. First-trimester prediction of pre-eclampsia: external validity of algorithms in a prospectively enrolled cohort. Ultrasound Obstet Gynecol 2014;44:279-85.

9. Magnussen EB, Vatten LJ, Smith GD, Romundstad PR. Hypertensive disorders in pregnancy and subsequently measured cardiovascular risk factors. Obstet Gynecol 2009;114:961-70.

10. Spaanderman M, Ekhart T, van Eyck J, et al. Preeclampsia and maladaptation to pregnancy: a role for atrial natriuretic peptide? Kidney Int 2001;60:1397-406.

11. Zandstra M, Stekkinger E, van der Vlugt MJ, et al. Cardiac diastolic dysfunction and metabolic syndrome in young women after placental syndrome. Obstet Gynecol 2010;115:101-8.

12. Forest JC, Girouard J, Masse J, et al. Early occurrence of metabolic syndrome after hypertension in pregnancy. Obstet Gynecol 2005;105:1373-80.

13. Stekkinger E, Zandstra M, Peeters LL, Spaanderman ME. Early-onset preeclampsia and the prevalence of postpartum metabolic syndrome. Obstet Gynecol 2009;114:1076-84.

14. Dekker GA, de Vries JI, Doelitzsch PM, et al. Underlying disorders associated with severe early-onset preeclampsia. Am J Obstet Gynecol 1995;173:1042-8.

15. Van Pampus MG, Dekker GA, Wolf H, et al. High prevalence of hemostatic abnormalities in women with a history of severe preeclampsia. Am J Obstet Gynecol 1999;180:1146-50.

16. Scholten RR, Hopman MT, Sweep FC, et al. Co-occurrence of cardiovascular and prothrombotic risk factors in women with a history of preeclampsia. Obstet Gynecol 2013;12:97-105.

17. Baschat AA, Magder LS, Doyle LE, et al. Prediction of preeclampsia utilizing the first trimester screening examination. Am J Obstet Gynecol 2014;211:514.e1-7.

18. Sibai B. First-trimester screening with combined maternal clinical factors, biophysical and biomarkers to predict preterm pre-eclampsia and hypertensive disorders: are they ready for clinical use? BJOG 2015;122:282-3.

19. Akolekar R, Syngelaki A, Poon L, et al. Competing risks model in early screening for preeclampsia by biophysical and biochemical markers. Fetal Diagn Ther 2013;33:8-15.

20. Audibert F, Boucoiran I, An N, et al. Screening for preeclampsia using first-trimester serum markers and uterine artery Doppler in nulliparous women. Am J Obstet Gynecol 2010;203:383.e1-8.

21. Poon L, Stratieva V, Piras S, et al. Hypertensive disorders in pregnancy: combined screening by uterine artery Doppler, blood pressure and serum PAPP-A at 11-13 weeks. Prenat Diagn 2010;30:216-23.

22. Park F, Leung C, Poon L, et al. Clinical evaluation of a first trimester algorithm predicting the risk of hypertensive disease of pregnancy. Aust N Z J Obstet Gynaecol 2013;53:532-9.

23. Scazzocchio E, Figueras F, Crispi F, et al. Performance of a firsttrimester screening of preeclampsia in a routine care low-risk setting. Am J Obstet Gynecol 2013;208:203e.1-10.

24. Odibo A, Zhong Y, Goetzinger K, et al. First-trimester placental protein 13, PAPP-A, uterine artery Doppler and maternal characteristics in the prediction of pre-eclampsia. Placenta 2011;32:598-02.
25. Kuc S, Koster M, Franx A, et al. Maternal characteristics. Mean arterial pressure and serum markers in early prediction of preeclampsia. Plos ONE 2013;8e63546.

26. Oliveira N, Doyle LE, Atlas RO, et al. External validity of first-trimester algorithms in the prediction of pre-eclampsia disease severity. Ultrasound Obstet Gynecol 2014;44:286-92.

27. Grundy SM, Brewer HB Jr, Cleeman JI, et al. American Heart Association; National Heart, Lung, and Blood Institute. Definition of metabolic syndrome: report of the National Heart, Lung, and Blood Institute/American Heart Association conference on scientific issues related to definition. Circulation 2004;109:433-8.

28. Villa PM, Kajantie E, Räikkönen K, et al. Aspirin in the prevention of pre-eclampsia in high-risk women: a randomized placebo-controlled PREDO Trial and a meta-analysis of randomized trials. BJOG 2013;120:64-74.

29. Zheng J, Shan PF, Gu W. The efficacy of metformin in pregnant women with polycystic ovary syndrome: a meta-analysis of clinical trials. J Endocrinol Invest 2013;36:797-802.

30. De Leo V, Musacchio MC, Piomboni P, et al. The administration of metformin during pregnancy reduces polycystic ovary syndrome related gestational complications. Eur J Obstet Gynecol Reprod Biol 2011;157:63-6.

31. Nawaz FH, Khalid R, Naru T, Rizvi J. Does continuous use of metformin throughout pregnancy improve pregnancy outcomes in women with polycystic ovarian syndrome? J Obstet Gynaecol Res 2008;34:832-7.

32. Salvesen KA, Vanky E, Carlsen SM. Metformin treatment in pregnant women with polycystic ovary syndrome-is reduced complication rate mediated by changes in the uteroplacental circulation? Ultrasound Obstet Gynecol 2007;29:433-7.

33. Fox KA, Longo M, Tamayo E, et al. Effects of pravastatin on mediators of vascular function in a mouse model of soluble Fms-like tyrosine kinase-1-induced preeclampsia. Am J Obstet Gynecol 2011;205: e1-366-e365.

34. Kumasawa K, Ikawa M, Kidoya $\mathrm{H}$, et al. Pravastatin induces placental growth factor and ameliorates preeclampsia in a mouse model. Proc Natl Acad Sci USA 2011;108:1451-5.

35. Bauer AJ, Banek CT, Needham K, et al. Pravastatin attenuates hypertension, oxidative stress, and angiogenic imbalance in rat model of placental ischemia-induced hypertension. Hypertension 2013;61:1103-10.

36. Saad AF, Kechichian T, Yin H, et al. Effects of pravastatin on angiogenic and placental hypoxic imbalance in a mouse model of preeclampsia. Reprod Sci 2014;21:138-45.

37. Gaillard R, Bakker R, Willemsen SP, et al. Blood pressure tracking during pregnancy and the risk of gestational hypertensive disorders: the Generation R Study. Eur Heart J 2011;32:3088-97.

38. Gillon TE, Pels A, von Dadelszen P, et al. Hypertensive disorders of pregnancy: a systematic review of international clinical practice guidelines. PLoS One 2014;9e113715.

39. Scantlebury DC, Schwartz GL, Acquah LA, et al. The treatment of hypertension during pregnancy: when should blood pressure medications be started? Curr Cardiol Rep 2013;15:412.

40. Cotter AM, Molloy AM, Scott JM, Daly SF. Elevated plasma homocysteine in early pregnancy: a risk factor for the development of severe pre-eclampsia. Am J Obstet Gynecol 2001;185:781-5. 